\title{
Global Analysis of an SEIRS Model for COVID-19 Capturing Saturated Incidence with Treatment Response
}

\author{
Oluyori, David A., $\quad$ Adebayo, Helen 0. \\ Department of Mathematics, School of Physical Science, \\ Ahmadu Bello University, Zaria, Kaduna State, Nigeria \\ oluyoridavid@gmail.com, helenlovejesus73@gmail.com \\ Pérez, Ángel G. C. \\ Facultad de Matemáticas, Universidad Autónoma de Yucatán, \\ Mérida, Yucatán, Mexico \\ agcp26@hotmail.com
}

\begin{abstract}
Sequel to V. A. Okhuese [Mathematical Predictions for COVID-19 as a Global Pandemic, medRxiv, 2020, https://doi.org/10.1101/2020.03.19.20038794], who studied the dynamics of COVID-19 using an SEIRUS model. We consider an SEIRS model capturing saturated incidence with treatment response. In this theoretical model, we assumed that the treatment response is proportional to the number of infected as long as the incidence cases are within the capacity of the healthcare system, after which the value becomes constant, when the number of confirmed cases exceed the carrying capacity of the available medical facilities. Thus, we obtain the reproduction number stating that when $R_{0}$ is less than a critical value $R_{1}$, the disease-free equilibrium is globally asymptotically stable. Also, we studied the existence of the local and global stability of the disease-free and endemic equilibria and found that the kind of treatment response and inhibitory measures deployed in tackling the COVID-19 pandemic determines whether the disease will die out or become endemic.
\end{abstract}

Keywords: COVID-19, SARS-CoV-2, Saturated Incidence, Treatment Function, Next Generation Matrix, Diseasefree and Endemic States, Local and Global Stability, Routh-Hurwitz Criterion, Lozinskiü measure, Lyapunov Function.

\section{Introduction}

The novel coronavirus disease (COVID-19) was first confirmed in the Chinese city of Wuhan, late December 2019. The rapidity of its spread in many countries around the globe made the WHO declare it as a global pandemic and public health emergency, raising concerns that if countries with robust healthcare systems to detect and control disease outbreak are having challenges managing the disease, countries with weak healthcare system need to put adequate measures in place to contain the spread [1]. The coronavirus disease (COVID-19) caused by the Severe Acute Respiratory Syndrome Coronavirus-2 (SARS-CoV-2) presents clinical features which are similar to the diseases caused by other coronaviruses, Severe Acute Respiratory Syndrome (SARS) and Middle East Respiratory Syndrome such as lower respiratory illness with 
fever, dry cough, myalgia, shortness of breath etc. The Coronavirus disease is "novel" in the sense that, it is a new strain of zoonotic origin which has not been previously discovered to affect humans. Historically, the COVID-19 pandemic is a major human coronavirus epidemic in the last two decades aside SARS [2] and MERS [3, 4] respectively. The incubation period of COVID-19 is between 2-14 days with symptoms averagely between 5-7 days. Its basic reproduction number is averaged 2.2 [5] and even more ranging from $1.4-6.5$ in [6]. Globally as at May $5^{\text {th }}, 2020$, there are 3,646,304 confirmed cases, 1,200,296 recovered and 252,425 deaths.

The SARS-CoV-2 is an enveloped positive-sense stranded RNA virus (ssRNA) (Subgenus: Sarbecovirus, Orthocoronavirinae subfamily), consisting of 29,903 nucleotides and two untranslated sequences of 254 and 229 nucleotides at the 5' and 3'-ends respectively (GenBank No. MN 908947) [7]. The coronaviruses $(\mathrm{CoV})$ are subdivided into 4 genera $\alpha-, \beta-, \gamma-, \delta-$ coronaviruses. The $\alpha-$ and $\beta-\mathrm{CoV}$ infect mammals while the $\gamma-$ and $\delta-\operatorname{CoV}$ infect birds. Previously, 6-CoVs have been identified as human susceptible virus, which are $\alpha-\operatorname{CoVs}$, $H C o V-229 E, H C o V-N L 63, \beta-C o V s, H C o V-H K U I$ and HCoV-OC43. Specifically, SARS-CoV-2 is of the $\beta$ - type, it enters the human epithelial via the binding of the viral spike protein $(S-$ Protein) to the human Angiotensin Converting Enzyme 2 (ACE-2), which is a functional receptor involved in arterial blood pressure control and fibrotic response to damage, using it as its binding site. [8,9]. Basically, there are 3 main transmission routes for the disease, which are: Droplets transmission, which occurs when respiratory droplets produced by infected persons when they cough or sneeze in close proximity with other people are inhaled or ingested; Contact transmission, which occur when people touch infected surfaces and subsequently touch their mouth, nose or eyes; Aerosol transmission, which occurs when respiratory droplets mix with the air, forming aerosols are inhaled in high doses, which are potent enough to cause infection [11].

\subsection{Models for incidence and treatment parameters}

According to [20], Incidence is the rate at which the susceptible become infectious. Thus, if the unit of time is days, then the incidence is the number of new infections per day. The classical Kermack-McKendrick model in [21] proposed a Bilinear Incidence rate for simple mass action denoted as $h(I)=\beta S I$, where $S$ and $I$ denote the susceptible and Infected respectively and $\beta$ denote the probability of transmission per contact. Next, we have Standard Incidence, which is denoted as $\frac{\beta S I}{N}$, where $N$ is the total population size, $\beta$ is the average number of effective contacts per unit time of an infective with the susceptible (daily contact rate), since $\frac{S}{N}$ is the susceptible fraction of the population, $\frac{\beta S I}{N}$ is the average number of infection transmissions by all infectives. According to literature, the bilinear and standard incidences have been extensively studied by various authors in [33 - 36] and others. Another kind of incidence that is of interest to our work is the Saturation incidence. In 1973, sequel to the study of cholera epidemic which occurred in 
Bari, Capasso and Serio [22] introduced the saturated incidence denoted as $h(I) S$ into epidemic models, where $h(I)$ tends to a saturation level when $I$ becomes large, i.e.,

$$
h(I) S=\frac{\beta I}{1+\alpha I} S \rightarrow \frac{\beta}{\alpha} S \text { as } I \rightarrow \infty \ldots \text { (1) }
$$

where $\beta I$ denotes the infection force of the disease and $\frac{I}{1+\alpha I}$ measures the inhibitory effect from the behavioural change of the susceptible when their number of incidence increases or from the crowding effect of the infective due to unrestrained contact using suitable parameters. Esteva and Matias [20] studied a model for disease transmitted by vector with saturating incidence such that the model assumes a saturating effect in the incidence due to the response of the vector to change in the susceptible and infected densities. The saturation incidence seems more realistic than the bilinear incidence due to the inclusion of behavioural change and crowding effect of the infective. In the face of the current realities from COVID-19, it is evident that we have high saturation incidence in which useful strategies need to be deployed to contain the spread through various interventions such as good hygiene, physical/social distancing, partial/total lockdown, travel/public gathering ban, good treatments, contact tracing, pool testing, etc, can help to reduce the high rate of secondary infections as stipulated by the WHO guidelines.

It is a general assumption in classical epidemic models that treatment rate of infection is assumed to be proportional to the number of the infective individuals and the recovery rate depends on the medical resources available such as test kits, drugs, isolation centres, ventilators, availability of trained medical personnel, efficiency of treatment. WHO situation reports from many nations have shown how stretched the healthcare systems of countries have been with its attendant high morbidity. Therefore, it is important for countries with increased cases to adopt suitable treatment functions. Wang and Ruan [23] introduced a constant treatment in SIR models as follows:

$$
T(I)=\left\{\begin{array}{ll}
r, & I>0 \\
0, & I=0
\end{array} \ldots . . .\right.
$$

where $r$ is a positive constant and $I$ is the number of infected individuals.

Recently, Wang [24] considered a piecewise linear treatment function defined as:

$$
T(I)=\left\{\begin{array}{cc}
r I, & 0 \leq I \leq I_{0} \\
k, & I>I_{0}
\end{array} \ldots \ldots . . .3\right)
$$

where $k=r I_{0}, r$ and $I_{0}$ are positive constants.

The first condition in (3) explains the proportionality of the treatment response to the number of the infectious people when the number of infectious is less than or equal to a fixed value $I_{0}$, the second typifies an endemic situation $\left(I>I_{0}\right)$, where the number of the infectious has increased to a saturation point where the available medical facilities are stretched beyond capacity and death toll rises in an unprecedented manner. Therefore, in many disease outbreaks there are 
different kinds of delays when they spread, such as latent period delay before symptoms surfaces and immunity period delay after recovery. Zhou [25] studied an SIR epidemic model with treatment function $h^{*}=\frac{\alpha I}{\omega+I}$. Zhang et'al [26] modified the model in [25], with saturated

incidence rate, $\frac{\beta S}{1+\alpha S}$ using the same treatment function. In [27] Agrawal et'al modified the work of [26] considering an SEIRS epidemic model with saturated incidence and treatment rate. Badole et'al in [28] taking some cue from [27] studied the global dynamics of an SEIR model with saturated incidence under treatment. Various authors have considered saturated incidence and treatment to study the stability and bifurcation of different dynamic systems in [24, 34, 37 39].

\subsection{Existing compartmental models for COVID-19}

Since the outbreak, many mathematical have appeared in an attempt to assess the dynamics of the COVID-19 epidemic. The first models were dynamic mechanistic models aimed at estimating of the basic reproduction number $R_{0}[12,13,14,15]$, also simple exponential growth models $[16,17]$. Other compartmental epidemiological models such as SIRD, SEIR, SEIRD and SEIRUS [21, 19, 18, 10] has been proposed to estimate other epidemiological parameters such as the transmission rate, local and global stability of the disease-free and endemic equilibria to provide insights for forecasting purposes.

Recently, [10] considered an SEIRUS (Susceptible-Exposed-Infected-Recovered-UndetectableSusceptible) model for COVID-19, where it was predicted that with strict adherence to the guidelines of the WHO on observatory and treatment procedures, the pandemic will soon die out. Based on the motivation from [10, 24, 27, 28, 29], we present an SEIRS (Susceptible-ExposedInfected-Recovered-Susceptible) model with saturated incidence and treatment functions which prescribes inhibitory measures such as personal hygiene, wearing of face mask, travel/public gathering ban, partial or total lockdown etc and rapid responses such as public enlightenment, pool testing, increased medical facilities and trained medical personnel etc, as potent means of slowing down the spread of COVID-19.

\section{Model description}

The model can be described as follows:

$$
\begin{gathered}
S^{\prime}(t)=A-\mu S-\frac{\beta S I}{1+\alpha I}+(\rho+\varepsilon) R \\
E^{\prime}(t)=\frac{\beta S I}{1+\alpha I}-(\gamma+\mu) E \ldots \ldots \ldots \\
I^{\prime}(t)=\gamma E-(\sigma+\mu+\varphi)-T(I)
\end{gathered}
$$




$$
\begin{gathered}
R^{\prime}(t)=\sigma I-(\rho+\varepsilon+\mu+\varpi) R+T(I) \\
N(t)=S(t)+E(t)+I(t)+R(t)
\end{gathered}
$$

Here, $S(t)$ is the number of susceptible per unit time, $E(t)$ is the number of the exposed per unit time, $I(t)$ is the number of the infectious per unit time, $R(t)$ is the number of the recovered per unit time, $A$ is the recruitment rate of the population, $\mu$ is the natural death rate of the population per time, $\rho$ is the recovery rate, $\alpha$ is the saturation parameter that measures the inhibitory effect, $\beta$ is the rate of transmission, $\varepsilon$ is the proportion of the removed population that is been observed and will subsequently move to the susceptible, $\gamma$ is the rate of developing infection/incidence rate. $\varphi$ is the disease-induced death rate of the infected population not quarantined, $\varpi$ is the fraction of the removed population under observation (the undetected) before moving to the susceptible class, $h(I) S=\frac{\beta S I}{1+\alpha I}$ is the saturation incidence parameter, $\frac{1}{1+\alpha I}$ is the inhibitory parameter and $T(I)$ is the treatment response as defined in (3). The flow diagram of the model can be seen in Figure 1.

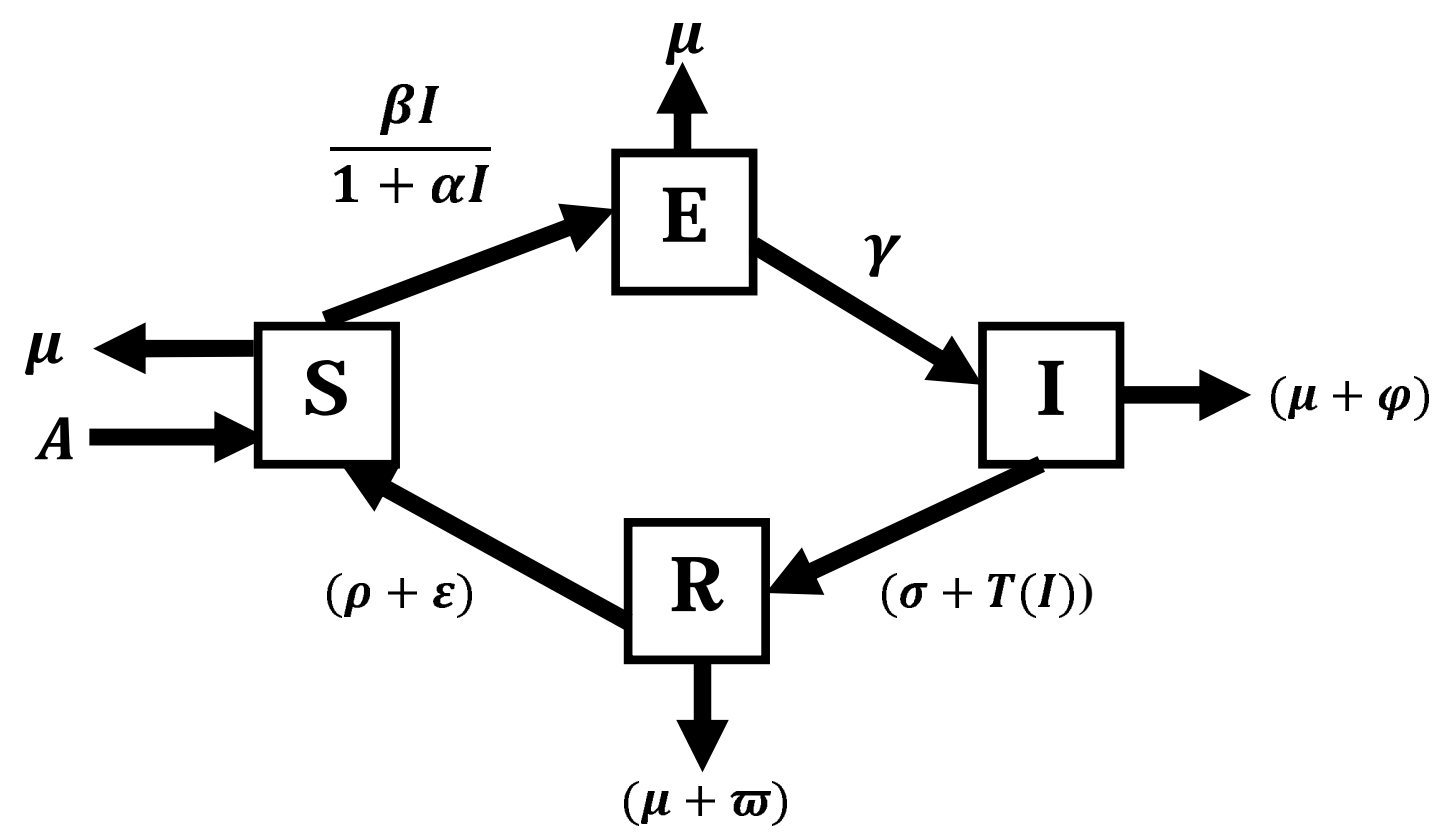

Figure 1. Schematic diagram of the model.

It follows from (4) that

$$
\begin{gathered}
(S+E+I+R)^{\prime}=A-\mu(S+E+I+R)-\varphi I-\varpi R \\
\leq A-\mu(S+E+I+R)
\end{gathered}
$$

Then lim $\sup _{n \rightarrow \infty}(S+E+I+R) \leq \frac{A}{\mu}$. The feasible region for system (4) is

$$
\Omega=\left\{(S+E+I+R): \limsup _{n \rightarrow \infty}(S+E+I+R) \leq \frac{A}{\mu}, S \geq 0, E \geq 0, I \geq 0, R \geq 0\right\} .
$$


Thus, it naturally follows that the region $\Omega$ is positively invariant with respect to system (4). Hence the system is mathematically and epidemiological well posed in $\Omega$.

\section{Dynamics near the disease-free equilibrium}

We will now study the local dynamics of system (4) near the disease-free equilibrium. Thus, we will focus here on the case when $0 \leq I \leq I_{0}$, that is, when $T(I)=r I$. In that case, system (4) becomes

$$
\begin{gathered}
S^{\prime}(t)=A-\mu S-\frac{\beta S I}{1+\alpha I}+(\rho+\varepsilon) R \\
E^{\prime}(t)=\frac{\beta S I}{1+\alpha I}-(\gamma+\mu) E \ldots \ldots \ldots \\
I^{\prime}(t)=\gamma E-(\sigma+\mu+\varphi) I-r I \\
R^{\prime}(t)=\sigma I-(\rho+\varepsilon+\mu+\varpi) R+r I .
\end{gathered}
$$

By simple calculation from the system (5), we obtain the equilibrium state where $S^{\prime}(t)=$ $E^{\prime}(t)=I^{\prime}(t)=R^{\prime}(t)=0$ (i.e. the LHS vanishes). Thus the steady state of system (5) satisfies the following algebraic system of equations:

$$
\begin{gathered}
S^{*}=\frac{(A+(\rho+\varepsilon) R)(1+\alpha I)}{\mu(1+\alpha I)+\beta I}, E^{*}=\frac{\beta I(A+(\rho+\varepsilon) R)}{(\gamma+\mu)(\mu(1+\alpha I)+\beta I)}, \\
I^{*}=\frac{\gamma \beta I(A+(\rho+\varepsilon) R-r)}{(\sigma+\mu+\varphi+r)(\gamma+\mu)(\mu(1+\alpha I)+\beta I)}, R^{*}=\frac{(\sigma+r) I}{(\rho+\varepsilon+\mu+\varpi)}
\end{gathered}
$$

At the disease-free equilibrium (DFE), when no disease outbreak occurs, no one is in the exposed or infected class and as such no one is in the recovered class. Therefore, $E=I=0$. On substitution, the algebraic system in (6) reduces to $S=\frac{A}{\mu}$.

Thus, we conclude the following result.

Lemma 3.1: The system (5) always has a disease-free equilibrium point $E_{0}=\left(\frac{A}{\mu}, 0,0,0\right)$.

\subsection{Basic reproduction number}

The basic reproduction number $R_{0}$ measures the probability of an infectious disease spreading through a population or becoming extinct after some time. This threshold characteristic of $R_{0}$ 
helps epidemiologist to make the following assumptions: (a) If $R_{0}<1$, the infection will die out with time and (b) If $R_{0}>1$, the disease will be endemic in the population.

Next, we find the reproduction number, $R_{0}$ of the system (4) by obtaining the Jacobian of the system and using the Next Generation Matrix due to Van den Driessche and Watmough [32].

The Jacobian of system (4) when $0 \leq I \leq I_{0}$ is given by

$$
J\left(S^{*}, E^{*}, I^{*}, R^{*}\right)=\left(\begin{array}{cccc}
-\left(\mu+\frac{\beta I}{1+\alpha I}\right) & 0 & \frac{\beta S}{(1+\alpha I)^{2}} & (\rho+\varepsilon) \\
\frac{\beta I}{1+\alpha I} & -(\gamma+\mu) & \frac{\beta S}{(1+\alpha I)^{2}} & 0 \\
0 & \gamma & -(\sigma+\mu+\varphi+r) & 0 \\
0 & 0 & (\sigma+r) & -(\rho+\varepsilon+\mu+\varpi)
\end{array}\right) \ldots(7)
$$

Applying the disease-free state condition $E_{0}=\left(\frac{A}{\mu}, 0,0,0\right)$ in the Jacobian matrix, we have

$$
J\left(\frac{A}{\mu}, 0,0,0\right)=\left[\begin{array}{cccc}
-\mu & 0 & \frac{\beta A}{\mu} & (\rho+\varepsilon) \\
\frac{\beta I}{1+\alpha I} & -(\gamma+\mu) & \frac{\beta A}{\mu} & 0 \\
0 & \gamma & -(\sigma+\mu+\varphi+r) & 0 \\
0 & 0 & (\sigma+r) & -(\rho+\varepsilon+\mu+\varpi)
\end{array}\right]
$$

Using the next generation matrices, it is clear that the reproduction number $R_{0}$ is the spectral radius of the next generation matrix derived from the exposed and infected class, i.e.,

$$
R_{0}=\rho(K)
$$

where $\rho(K)$ is the spectral radius and $K=F V^{-1}$ is the next generation matrix. $F$ is derived from the exposed and infected class and $V$ are the remaining terms after $F$ is taken.

Thus

$$
\begin{gathered}
F=\left[\begin{array}{ll}
0 & \frac{\beta A}{\mu} \\
0 & 0
\end{array}\right] \\
V=\left[\begin{array}{cc}
-(\gamma+\mu) & 0 \\
\gamma & -(\sigma+\mu+\varphi+r)
\end{array}\right] \\
V^{-1}=\left[\begin{array}{cc}
(\gamma+\mu) & 0 \\
-\gamma & (\sigma+\mu+\varphi+r)
\end{array}\right]
\end{gathered}
$$




$$
\begin{aligned}
K=F V^{-1} & =\left[\begin{array}{cc}
0 & \frac{\beta A}{\mu} \\
0 & 0
\end{array}\right]\left[\begin{array}{cc}
(\gamma+\mu) & 0 \\
-\gamma & (\sigma+\mu+\varphi+r)
\end{array}\right] \\
= & {\left[\begin{array}{cc}
\frac{\gamma \beta A}{\mu(\mu+\gamma)(\sigma+\mu+\varphi+r)} & \frac{\beta A}{\mu(\mu+\gamma)(\sigma+\mu+\varphi+r)} \\
0 & 0
\end{array}\right] }
\end{aligned}
$$

$K$ is the next generation matrix of the system (4).

The spectral radius is

$$
\rho(K)=\frac{\gamma \beta A}{\mu(\mu+\gamma)(\sigma+\mu+\varphi+r)}
$$

Hence the basic reproduction number $R_{0}$ of system (4) is

$$
R_{0}=\frac{\gamma \beta A}{\mu(\mu+\gamma)(\sigma+\mu+\varphi+r)}
$$

\subsection{Local stability analysis of the disease-free equilibrium}

We examined the local stability of the DFE by the analysis of the eigenvalues of the Jacobian matrices of (4) at the equilibrium using the Routh-Hurwitz Criterion.

Theorem 3.2: The disease-free equilibrium $\left(E^{0}\right)$ is

(a) Locally asymptotically stable if $R_{0}<1$.

(b) Unstable if $R_{0}>1$.

Proof: The Jacobian matrix of the system (4) at the disease-free equilibrium is

$$
E^{0}=J\left(\frac{A}{\mu}, 0,0,0\right)
$$

$$
=\left[\begin{array}{cccc}
-\mu & 0 & \frac{\beta A}{\mu} & (\rho+\varepsilon) \\
0 & -(\gamma+\mu) & \frac{\beta A}{\mu} & 0 \\
0 & \gamma & -(\sigma+\mu+\varphi+r) & 0 \\
0 & 0 & (\sigma+r) & -(\rho+\varepsilon+\mu+\varpi)
\end{array}\right] .
$$

The characteristic equation of the system (5) at $E^{0}$ is

$$
\begin{gathered}
(\mu+\lambda)(\sigma+\mu+\varphi+r+\lambda)\left[\lambda^{2}+(\gamma+\sigma+\mu+\varphi+r+2 \mu) \lambda+(\sigma+\mu+\varphi+r)(\gamma+\mu)\right. \\
\left.-\frac{\gamma \beta A}{\mu}\right]=0 . \quad \ldots \quad \text { (11) }
\end{gathered}
$$


By (11) it is clear that $\lambda_{1}=-\mu$ and $\lambda_{2}=-(\sigma+\mu+\varphi+r)$ are two roots of (11). The other roots of (11) are determined by the equation

$$
\lambda^{2}+(\gamma+\sigma+\mu+\varphi+r+2 \mu) \lambda+(\sigma+\mu+\varphi+r)(\gamma+\mu)-\frac{\gamma \beta A}{\mu}=0,
$$

which has negative roots if and only if $(\sigma+\mu+\varphi+r)(\gamma+\mu)-\frac{\gamma \beta A}{\mu}>0$, which is equivalent to the reproduction number $R_{0}$ being less than one. This implies that the disease-free equilibrium $E^{0}$ is locally asymptotically stable when $R_{0}<1$ and unstable when $R_{0}>1$.

\section{Existence of endemic equilibria}

In this section, we consider the endemic equilibria of system (4). An endemic equilibrium of (4) satisfies the system

$$
\begin{gathered}
E=\frac{(\sigma+\mu+\varphi) I+T(I)}{\gamma}, \\
R=\frac{\sigma I+T(I)}{\rho+\varepsilon+\mu+\varpi}, \\
S=\frac{(1+\alpha I)(\gamma+\mu) E}{\beta I}, \\
A-\mu S+(\rho+\varepsilon) R-(\gamma+\mu) E=0 .
\end{gathered}
$$

Suppose that $P^{*}\left(S^{*}, E^{*}, I^{*}, R^{*}\right)$ is an endemic equilibrium of (4). If $0<I^{*} \leq I_{0}$, then $P^{*}$ satisfies

$$
\begin{gathered}
E^{*}=\frac{(\sigma+\mu+\varphi+r) I^{*}}{\gamma}, \\
R^{*}=\frac{(\sigma+r) I^{*}}{\rho+\varepsilon+\mu+\varpi}, \\
S^{*}=\frac{\left(1+\alpha I^{*}\right)(\gamma+\mu)(\sigma+\mu+\varphi+r)}{\beta \gamma}
\end{gathered}
$$

and

$$
\begin{aligned}
& I^{*} \\
& =\frac{\mu(\mu+\gamma)(\sigma+\mu+\varphi+r)(\rho+\varepsilon+\mu+\varpi)\left(R_{0}-1\right)}{\alpha \mu(\mu+\gamma)(\rho+\varepsilon+\mu+\varpi) r+\beta(\mu+\gamma)(\rho+\varepsilon+\mu+\varpi)(\sigma+\mu+\varphi+r)-\beta \gamma(\rho+\varepsilon)(\sigma+r)} .
\end{aligned}
$$

The denominator of the expression for $I^{*}$ is always positive, while the numerator is positive if and only if $R_{0}>1$. The condition $I^{*} \leq I_{0}$ is equivalent to $R_{0} \leq 1+\frac{q_{0}}{p_{0}} I_{0}$, where

$$
\begin{gathered}
p_{0}=\mu(\mu+\gamma)(\sigma+\mu+\varphi+r)(\rho+\varepsilon+\mu+\varpi), \\
q_{0}=\alpha \mu(\mu+\gamma)(\rho+\varepsilon+\mu+\varpi) r+\beta(\mu+\gamma)(\rho+\varepsilon+\mu+\varpi)(\sigma+\mu+\varphi+r) \\
-\beta \gamma(\rho+\varepsilon)(\sigma+r) .
\end{gathered}
$$


Therefore, we can conclude the following result.

Theorem 4.1: $P^{*}\left(S^{*}, E^{*}, I^{*}, R^{*}\right)$ is an endemic equilibrium of (4) if and only if

where $p_{0}$ and $q_{0}$ are given by (12).

$$
1<R_{0} \leq 1+\frac{q_{0}}{p_{0}} I_{0}
$$

Next, we study the endemic equilibria of (4) when $I>I_{0}$ and hence $T(I)=k$.

An endemic equilibrium $(S, E, I, R)$ with $I>I_{0}$ satisfies

$$
\begin{gathered}
E=\frac{(\sigma+\mu+\varphi) I+k}{\gamma}, \\
R=\frac{\sigma I+k}{\rho+\varepsilon+\mu+\varpi}, \\
S=\frac{(1+\alpha I)(\gamma+\mu)((\sigma+\mu+\varphi) I+k)}{\beta \gamma I} .
\end{gathered}
$$

Substituting these expressions in the equation $A-\mu S+(\rho+\varepsilon) R-(\gamma+\mu) E=0$, we obtain the quadratic equation

$$
A_{1} I^{2}+B_{1} I+C_{1}=0
$$

where

$$
\begin{gathered}
A_{1}=(\alpha \mu+\beta)(\rho+\varepsilon+\mu+\varpi)(\sigma+\mu+\varphi)(\mu+\gamma)-\beta(\rho+\varepsilon) \sigma \gamma>0, \\
B_{1}=(\alpha \mu k+\beta k+\mu)(\rho+\varepsilon+\mu+\varpi)(\mu+\gamma)-\beta \gamma A(\rho+\varepsilon+\mu+\varpi)-\beta \gamma k(\rho+\varepsilon), \\
C_{1}=\mu k(\rho+\varepsilon+\mu+\varpi)(\mu+\gamma)>0 .
\end{gathered}
$$

If $B_{1} \geq 0$, it is clear that (13) does not have any positive solutions. Consider the case when $B_{1}<0$. Let $\Delta=B_{1}{ }^{2}-4 A_{1} C_{1}$. The solutions are given by

$$
I_{1}=\frac{-B_{1}-\sqrt{\Delta}}{2 A_{1}}, \quad I_{2}=\frac{-B_{1}+\sqrt{\Delta}}{2 A_{1}} .
$$

These solutions are positive and distinct only when $\Delta>0$. The two solutions coalesce into a double positive root when $\Delta=0$. Lastly, (13) has no real solutions when $\Delta<0$. Hence, we obtain the following result.

Theorem 4.2: Let $A_{1}, B_{1}$ and $C_{1}$ be given by (14). Define $\Delta=B_{1}{ }^{2}-4 A_{1} C_{1}$,

and

$$
I_{1}=\frac{-B_{1}-\sqrt{\Delta}}{2 A_{1}}, \quad I_{2}=\frac{-B_{1}+\sqrt{\Delta}}{2 A_{1}}
$$

$$
\begin{gathered}
S_{i}=\frac{\left(1+\alpha I_{i}\right)(\gamma+\mu)\left((\sigma+\mu+\varphi) I_{i}+k\right)}{\beta \gamma I_{i}}, \quad E_{i}=\frac{(\sigma+\mu+\varphi) I_{i}+k}{\gamma}, \\
R_{i}=\frac{\sigma I_{i}+k}{\rho+\varepsilon+\mu+\varpi}
\end{gathered}
$$


for $i=1,2$. Then $P_{i}\left(S_{i}, E_{i}, I_{i}, R_{i}\right)$ is an endemic equilibrium of (4) if and only if $B_{1}<0, \Delta \geq 0$ and $I_{i}>I_{0}$. Moreover, $P_{1}$ and $P_{2}$ coalesce into a single endemic equilibrium when $B_{1}<0$, $\Delta=0$ and $I_{1}=I_{2}>I_{0}$.

\section{Global stability of the disease-free equilibrium}

In this section, we analyze the global stability of the disease-free equilibrium for system (4) using the method of Lyapunov functions.

Theorem 5.1: Let

$$
R_{1}:=1-\frac{r}{\sigma+\mu+\varphi+r}
$$

If $R_{0}<R_{1}$, then the disease-free state equilibrium $E^{0}$ is globally asymptotically stable.

Proof: From the system of equations in (5), we have $\frac{d S}{d t} \leq A-\mu S$. A solution of the equation $\frac{d y}{d t} \leq A-\mu y$ is a maximal solution of $S(t)$. We recall that $y \rightarrow \frac{A}{\mu}$ as $t \rightarrow \infty$. By applying the comparison theorem, we obtain $S(t) \leq \frac{A}{\mu}$, also from the set

$$
\Omega=\left\{(S+E+I+R): S+E+I+R \leq \frac{A}{\mu}, S \geq 0, E \geq 0, I \geq 0, R \geq 0\right\}
$$

we have $I(t) \leq \frac{A}{\mu}$.

Define the Lyapunov function

$$
L=\gamma E-(\gamma+\mu) I
$$

From $R_{0}<1-\frac{r}{\sigma+\mu+\varphi+r}$, we have $\frac{\gamma \beta A}{\mu(\mu+\gamma)(\sigma+\mu+\varphi+r)}<\frac{\sigma+\mu+\varphi}{\sigma+\mu+\varphi+r}$, and then $\gamma \beta\left(\frac{A}{\mu}\right)-(\gamma+\mu)(\sigma+$ $\mu+\varphi)<0$.

We have thus $L^{\prime}=\gamma E^{\prime}-(\gamma+\mu) I^{\prime}$, that is,

$$
\begin{aligned}
L^{\prime} & =\gamma\left[\frac{\beta S I}{1+\alpha I}-(\gamma+\mu) E\right]+(\gamma+\mu)[\gamma E-(\sigma+\mu+\varphi) I+T(I)] \\
& =\left[\frac{\gamma \beta S}{1+\alpha I}-(\gamma+\mu)(\sigma+\mu+\varphi)\right] I-(\gamma+\mu) T(I) \\
& \leq[\gamma \beta S-(\gamma+\mu)(\sigma+\mu+\varphi+r)] I
\end{aligned}
$$

Recall that $S \leq \frac{A}{\mu}$. Therefore,

$$
L^{\prime} \leq\left[\gamma \beta\left(\frac{A}{\mu}\right)-(\gamma+\mu)(\sigma+\mu+\varphi)\right] I \leq 0
$$


and $L^{\prime}=0$ if and only if $I=0$. Thus, the largest compact invariant set in $\left\{(S, E, I, R) \in \Omega, L^{\prime}=\right.$ $0\}$ is the singleton $E^{0}$. Therefore, by Lasalle-Lyapunov theorem, every solution that starts in $\Omega$ approaches $E^{0}$ as $t \rightarrow \infty$ and the proof is complete.

\section{Local stability of endemic equilibria}

We consider the local stability of the endemic equilibrium point $P^{*}\left(S^{*}, E^{*}, I^{*}, R^{*}\right)$ by analyzing the eigenvalues of the Jacobian matrices of (4) at the endemic equilibrium point using the RouthHurwitz Criterion.

Theorem 6.1: Let $P^{*}\left(S^{*}, E^{*}, I^{*}, R^{*}\right)$ be an endemic equilibrium of (4) with $I^{*} \leq I_{0}$. Then $P^{*}$ is locally asymptotically stable if and only if $a_{3}>0, a_{4}>0$ and $a_{1} a_{2} a_{3}>a_{3}{ }^{2}+a_{1}{ }^{2} a_{4}$, where $a_{1}, a_{2}, a_{3}$ and $a_{4}$ are given in (17).

Proof: When $I^{*} \leq I_{0}$, system (4) can be written as (5). The Jacobian of system (5) at the endemic state $P^{*}$ is

$$
\begin{aligned}
& J\left(S^{*}, E^{*}, I^{*}, R^{*}\right) \\
& =\left(\begin{array}{ccccc}
-\left(\mu+\frac{\beta I^{*}}{1+\alpha I^{*}}\right) & & 0 & \frac{\beta S^{*}}{\left(1+\alpha I^{*}\right)^{2}} & (\rho+\varepsilon) \\
\frac{\beta I^{*}}{1+\alpha I^{*}} & & -(\gamma+\mu) & \frac{\beta S^{*}}{\left(1+\alpha I^{*}\right)^{2}} & 0 \\
0 & \gamma & -(\sigma+\mu+\varphi+r) & 0 \\
0 & 0 & (\sigma+r) & -(\rho+\varepsilon+\mu+\varpi)
\end{array}\right),
\end{aligned}
$$

from which we obtain the characteristic equation

$$
\lambda^{4}+a_{1} \lambda^{3}+a_{2} \lambda^{2}+a_{3} \lambda+a_{4}=0
$$

where $a_{1}, a_{2}, a_{3}$, and $a_{4}$ are as defined below:

$$
\begin{aligned}
& a_{1}=(\gamma+\sigma+\varphi+r+\rho+\varepsilon+\varpi+4 \mu)+\frac{\beta I^{*}}{1+\alpha I^{*}}, \\
& a_{2}=(\rho+\varepsilon+\mu+\varpi)\left(\mu+\frac{\beta I^{*}}{1+\alpha I^{*}}\right)+(\gamma+\mu)\left(\rho+\varepsilon+\varpi+2 \mu+\frac{\beta I^{*}}{1+\alpha I^{*}}\right)+(\sigma+\mu+\varphi+ \\
& r)\left(\gamma+\rho+\varepsilon+\varpi+3 \mu+\frac{\beta I^{*}}{1+\alpha I^{*}}\right)-\frac{\gamma \beta S^{*}}{\left(1+\alpha I^{*}\right)^{2}},
\end{aligned}
$$




$$
\begin{aligned}
& a_{3}=\left(\mu+\frac{\beta I^{*}}{1+\alpha I^{*}}\right)[(\rho+\varepsilon+\mu+\varpi)(\gamma+\sigma+\varphi+r+2 \mu)]+(\gamma+\mu)(\rho+\varepsilon+\varpi+2 \mu+ \\
& \left.\frac{\beta S^{*}}{\left(1+\alpha I^{*}\right)^{2}}\right)(\sigma+\mu+\varphi+r)-\frac{\gamma \beta S^{*}}{\left(1+\alpha I^{*}\right)^{2}}(\rho+\varepsilon+\varpi+2 \mu) \\
& a_{4}=\left(\mu+\frac{\beta I^{*}}{1+\alpha I^{*}}\right)(\gamma+\mu)(\rho+\varepsilon+\mu+\varpi)(\sigma+\mu+\varphi+r) \\
& +\frac{\gamma \beta}{1+\alpha I^{*}}\left[\frac{(\rho+\varepsilon+\mu+\varpi) S^{*} I^{*}}{\left(1+\alpha I^{*}\right)^{2}}\right. \\
& \left.-\left\{\frac{(\rho+\varepsilon+\mu+\varpi) S^{*}}{1+\alpha I^{*}}\left(\mu+\frac{\beta I^{*}}{1+\alpha I^{*}}\right)+\rho(\gamma+\mu) I^{*}\right\}\right] .
\end{aligned}
$$

Notice that $a_{1}$ is always positive. Thus, by the Routh-Hurwitz criterion, we have that the endemic equilibrium $P^{*}\left(S^{*}, E^{*}, I^{*}, R^{*}\right)$ of (4) is locally asymptotically stable if and only if $a_{3}>0, a_{4}>0$ and $a_{1} a_{2} a_{3}>a_{3}{ }^{2}+a_{1}{ }^{2} a_{4}$. Hence, the theorem follows.

Next, we discuss the local stability for the second case of the treatment function, that is, when $T(I)=k$, which corresponds to an equilibrium with $I^{*}>I_{0}$. In this case, we have the system

$$
\begin{gathered}
S^{\prime}(t)=A-\mu S-\frac{\beta S I}{1+\alpha I}+(\rho+\varepsilon) R \\
E^{\prime}(t)=\frac{\beta S I}{1+\alpha I}-(\gamma+\mu) E \ldots \ldots \ldots \\
I^{\prime}(t)=\gamma E-(\sigma+\mu+\varphi) I-k \\
R^{\prime}(t)=\sigma I-(\rho+\varepsilon+\mu+\varpi) R+k .
\end{gathered}
$$

Theorem 6.2: Let $P^{*}\left(S^{*}, E^{*}, I^{*}, R^{*}\right)$ be an endemic equilibrium of (4) with $I^{*}>I_{0}$. Then $P^{*}$ is locally asymptotically stable if and only if $b_{3}>0, b_{4}>0$ and $b_{1} b_{2} b_{3}>b_{3}{ }^{2}+b_{1}{ }^{2} b_{4}$, where $b_{1}, b_{2}, b_{3}$ and $b_{4}$ are given in (21).

Proof: The Jacobian matrix of system (18) at $P^{*}$ is

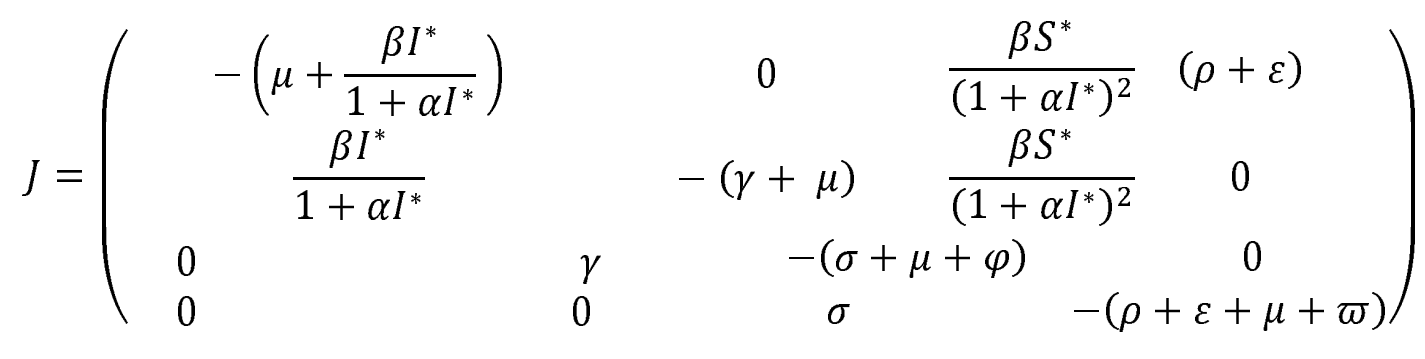

from which we obtain the characteristic equation 


$$
\lambda^{4}+b_{1} \lambda^{3}+b_{2} \lambda^{2}+b_{3} \lambda+b_{4}=0
$$

where $b_{1}, b_{2}, b_{3}$, and $b_{4}$ are as defined below:

$$
\begin{aligned}
& b_{1}=(\gamma+\sigma+\varphi+\rho+\varepsilon+\varpi+4 \mu)+\frac{\beta I^{*}}{1+\alpha I^{*}} \\
& b_{2}=(\rho+\varepsilon+\mu+\varpi)\left(\mu+\frac{\beta I^{*}}{1+\alpha I^{*}}\right)+(\gamma+\mu)\left(\rho+\varepsilon+\varpi+2 \mu+\frac{\beta I^{*}}{1+\alpha I^{*}}\right)+(\sigma+\mu+\varphi)(\gamma+ \\
& \left.\rho+\varepsilon+\varpi+3 \mu+\frac{\beta I^{*}}{1+\alpha I^{*}}\right)-\frac{\gamma \beta S^{*}}{\left(1+\alpha I^{*}\right)^{2}} \\
& b_{3}=\left(\mu+\frac{\beta I^{*}}{1+\alpha I^{*}}\right)[(\rho+\varepsilon+\mu+\varpi)(\gamma+\sigma+\varphi+2 \mu)]+(\gamma+\mu)(\rho+\varepsilon+\varpi+2 \mu+ \\
& \left.\begin{array}{c}
\beta S^{*} \\
\left(1+\alpha I^{*}\right)^{2}
\end{array}\right)(\sigma+\mu+\varphi)-\frac{\gamma \beta S^{*}}{\left(1+\alpha I^{*}\right)^{2}}(\rho+\varepsilon+\varpi+2 \mu) \\
& b_{4}=\left(\mu+\frac{\beta I^{*}}{1+\alpha I^{*}}\right)(\gamma+\mu)(\rho+\varepsilon+\mu+\varpi)(\sigma+\mu+\varphi) \\
& \quad+\frac{\gamma \beta}{1+\alpha I^{*}}\left[\frac{(\rho+\varepsilon+\mu+\varpi) S^{*} I^{*}}{\left(1+\alpha I^{*}\right)^{2}}\right. \\
& \left.\quad-\left\{\frac{(\rho+\varepsilon+\mu+\varpi) S^{*}}{1+\alpha I^{*}}\left(\mu+\frac{\beta I^{*}}{1+\alpha I^{*}}\right)+\rho(\gamma+\mu) I^{*}\right\}\right]
\end{aligned}
$$

It is clear that $b_{1}$ is always positive. Thus, by the Routh-Hurwitz criterion, we have that the endemic equilibrium $P^{*}\left(S^{*}, E^{*}, I^{*}, R^{*}\right)$ of (4) is locally asymptotically stable if and only if $b_{3}>0, b_{4}>0$ and $b_{1} b_{2} b_{3}>{b_{3}}^{2}+b_{1}{ }^{2} b_{4}$. Hence, the theorem follows.

\section{Global stability of endemic equilibria}

In this following, we analyze the global stability of the endemic equilibrium when $I^{*} \leq I_{0}$. To do this, we reduce the system of equations in (5) using $R(t)=\frac{A}{\mu}-S(t)-E(t)-I(t)$ to eliminate the $R(t)$ component from the first equation of system (5) to obtain a three-dimensional system given below: 


$$
\begin{gathered}
S^{\prime}(t)=\frac{A}{\mu}-\mu E-\frac{\beta S I}{1+\alpha I}+(\sigma+\mu+\varphi-r) I \\
E^{\prime}(t)=\frac{\beta S I}{1+\alpha I}-(\gamma+\mu) E \quad \ldots \ldots \quad(22) \\
I^{\prime}(t)=\gamma E-(\sigma+\mu+\varphi+r) I
\end{gathered}
$$

with initial conditions $S \geq 0, E \geq 0, I \geq 0$.

We consider the geometric approach due to Li and Muldowney [31] to obtain the global stability of the endemic equilibrium and find the sufficient conditions for which the endemic equilibrium is globally asymptotically stable. We describe the geometric approach method as follows.

We consider

$$
x^{\prime}=f(x)
$$

where $f: D \rightarrow R^{n}, D \subset R^{n}$ is a simply connected open set and $f \in C^{1}(D)$.

Let $x^{*}$ be an equilibrium of the equation (23), i.e., $f\left(x^{*}\right)=0$. Assume that the following hypotheses hold.

$(Y I)$ : There exists a compact absorbing set $K \subset D$.

(Y2): Equation (23) has a unique equilibrium $x^{*} \in D$.

The basic idea in this method is that if the equilibrium $x^{*}$ is locally stable, then the stability will hold provided the conditions in $(Y 1)$ and $(Y 2)$ are satisfied and no non-constant periodic solution of (23) exists. Thus, the sufficient conditions on $f$ capable of precluding the existence of such solutions must be found.

Suppose that the conditions (Y1) and (Y2) is satisfied. Assume that (23) satisfies a Bendixson criterion that is robust under $C^{1}$ local perturbations of $f$ at all non-equilibrium non-wandering points for (23). Then $x^{*}$ is globally stable in $D$ provided it is stable. Let $P(x)$ be an $\left(\begin{array}{l}n \\ 2\end{array}\right) \times\left(\begin{array}{l}n \\ 2\end{array}\right)$ matrix valued function that is $C^{1}$ on $D$ and consider

$$
B=P_{f} P^{-1}+P \frac{\partial F^{[2]}}{\partial x} P^{-1}
$$

where the matrix $P_{f}$ is

$$
\frac{\partial P_{i j}^{*}}{\partial x} f=\left.\frac{d P_{i j}}{d t}\right|_{(23)} \ldots \ldots \ldots
$$

and the matrix $J^{[2]}$ is the second additive compound matrix of the Jacobian matrix $J$, that is, $J(x)=D f(x)$. Generally speaking, for an $n \times n$ matrix $J=\left(J_{i j}\right), J^{[2]}$ is an $\left(\begin{array}{l}n \\ 2\end{array}\right) \times\left(\begin{array}{l}n \\ 2\end{array}\right)$ matrix and in the special case $n=3$ one has 


$$
J^{[2]}=\left(\begin{array}{ccc}
J_{11}+J_{22} & J_{23} & -J_{13} \\
J_{32} & J_{11}+J_{33} & J_{12} \\
-J_{31} & J_{21} & J_{22}+J_{33}
\end{array}\right) \cdot \cdots
$$

Consider the Lozinskiı̌ measure $\mu$ of $B$ with respect of a vector norm $\|\cdot\|$ in $R^{N}, N=\left(\begin{array}{l}n \\ 2\end{array}\right)$, defined by

$$
\mu(B)=\lim _{h \rightarrow 0^{+}} \frac{\|I+h B\|-1}{h} \ldots \ldots . .
$$

Suppose that $(Y 1)$ and $(Y 2)$ hold and consider the condition

$$
q:=\limsup _{t \rightarrow \infty} \sup _{x_{0} \in K} \frac{1}{t} \int_{0}^{t} \mu\left(B\left(x\left(s, x_{0}\right)\right)\right) d s<0 . \quad \ldots \quad \ldots
$$

It is shown in [31] that, if $D$ is simply connected, the condition $q<0$ rules out the presence of any orbit that gives rise to a simple closed rectifiable curve that is invariant for (23), such as periodic orbits, homoclinic orbits and heteroclinic cycles. Moreover, it is robust under $C^{\mathbf{1}}$ local perturbations of $f$ near any non-equilibrium point that is non-wandering. In particular, the following global stability result is proved in [31].

Lemma 7.1: Assume that $D$ is simply connected and that the assumptions $(Y 1)$ and $(Y 2)$ is satisified. Then the unique equilibrium $x^{*}$ of (23) is globally stable in $D$ if $q<0$.

Having established the above, we study the global stability of the endemic equilibrium $P^{*}$ and obtain the following result.

Theorem 7.2 Let $P^{*}\left(S^{*}, E^{*}, I^{*}, R^{*}\right)$ be an endemic equilibrium of (4) with $I^{*} \leq I_{0}$. If $\mu-\gamma>0$ then the endemic equilibrium $P^{*}$ is globally stable.

Proof: Since $I^{*} \leq I_{0},\left(S^{*}, E^{*}, I^{*}\right)$ is an equilibrium for system (22). The Jacobian matrix of system (22) is given as

$$
J=\left(\begin{array}{ccc}
-\frac{\beta S I}{1+\alpha I} & -\mu & -\frac{\beta S}{(1+\alpha I)^{2}}+(\sigma+\varphi+r-\mu) \\
\frac{\beta S I}{1+\alpha I} & -(\gamma+\mu) & \frac{\beta S}{(1+\alpha I)^{2}} \\
0 & \gamma & -(\sigma+\mu+\varphi+r)
\end{array}\right) \ldots \ldots
$$

and its second additive matrix is 


$$
J^{[2]}=\left(\begin{array}{ccc}
-\left(\gamma+\mu+\frac{\beta S I}{1+\alpha I}\right) & \frac{\beta S}{(1+\alpha I)^{2}} & \frac{\beta S}{(1+\alpha I)^{2}}-(\sigma+\varphi+r-\mu) \\
\gamma & -\left(\sigma+\mu+\varphi+r+\frac{\beta S I}{1+\alpha I}\right) & -\mu \\
0 & \frac{\beta S I}{1+\alpha I} & -(\gamma+\sigma+\varphi+r+2 \mu)
\end{array}\right)
$$

Choose the function $P=P(S, E, I)=\operatorname{diag}\left(1, \frac{E}{I}, \frac{E}{I}\right) ;$ then $P^{-1}=\operatorname{diag}\left(1, \frac{I}{E}, \frac{I}{E}\right)$ and

$$
P_{f}=\operatorname{diag}\left(0, \frac{E^{\prime} I-I^{\prime} E}{I^{2}}, \frac{E^{\prime} I-I^{\prime} E}{I^{2}}\right)
$$

Then we have

$$
\begin{gathered}
P_{f} P^{-1}=\operatorname{diag}\left(0, \frac{E^{\prime}}{E}-\frac{I^{\prime}}{I}, \frac{E^{\prime}}{E}-\frac{I^{\prime}}{I}\right) \\
P J^{[2]} P^{-1}=\left(\begin{array}{ccc}
-(\gamma+\mu)-\frac{\beta S I}{1+\alpha I} & \frac{\beta S I}{(1+\alpha I)^{2} E} & \frac{\beta S I}{(1+\alpha I)^{2} E}-(\sigma+\varphi+r-\mu) \frac{I}{E} \\
\frac{\gamma E}{I} & -(\sigma+\mu+\varphi+r)-\frac{\beta S I}{1+\alpha I} & -\mu \\
0 & \frac{\beta S I}{1+\alpha I} & -(\gamma+\sigma+\varphi+r+2 \mu)
\end{array}\right) \ldots
\end{gathered}
$$

The matrix $B=P_{f} P^{-1}+P J^{[2]} P^{-1}$ can be written in matrix form as

Where

$$
B=\left(\begin{array}{ll}
B_{11} & B_{12} \\
B_{21} & B_{22}
\end{array}\right) \ldots
$$

$$
\begin{array}{cc}
B_{11}=-(\gamma+\mu)-\frac{\beta S I}{1+\alpha I}, & B_{12}=\left(\frac{\beta S I}{(1+\alpha I)^{2} E}, \frac{\beta S I}{(1+\alpha I)^{2} E}-(\sigma+\varphi+r-\mu) \frac{I}{E}\right) \\
B_{21}=\left(\frac{\gamma E}{I}, 0\right)^{T}, & \\
B_{22}=\left(\begin{array}{cc}
-(\sigma+\mu+\varphi+r)-\frac{\beta S I}{1+\alpha I}+\frac{E^{\prime}}{E}-\frac{I^{\prime}}{I} & -\mu \\
\frac{\beta S I}{1+\alpha I} & -(\gamma+\sigma+\varphi+r+2 \mu)+\frac{E^{\prime}}{E}-\frac{I^{\prime}}{I}
\end{array}\right)
\end{array}
$$

Let $(u, v, w)$ be a vector in $\mathbb{R}^{3}$, its norm $\|\cdot\|$ is defined as

$$
\|(u, v, w)\|=\max \{|u|,|v|+|w|\} \ldots . .(34 n)
$$

Let $\mu(B)$ be the Lozinskir measure with respect to this norm. Thus, we have

$$
\mu(B) \leq \sup \left\{g_{1}, g_{2}\right\}
$$

where $g_{1}=B_{11}+\left|B_{12}\right|$ and $g_{2}=\mu_{2}\left(B_{22}\right)+\left|B_{21}\right| ;\left|B_{12}\right|$ and $\left|B_{21}\right|$ are matrix norms with respect to $l_{1}$ vector norm and $\mu_{2}$ denotes the Lozinskiĭ measure with respect to $l_{1}$ vector norm. Then, 


$$
\begin{gathered}
\mu_{1}\left(B_{11}\right)=-(\gamma+\mu)-\frac{\beta S I}{1+\alpha I} \ldots . .(35) \\
\left|B_{12}\right|=\max \left(\frac{\beta S I}{(1+\alpha I)^{2} E}, \quad \frac{\beta S I}{(1+\alpha I)^{2} E}-(\sigma+\varphi+r-\mu) \frac{I}{E}\right)=\frac{\beta S I}{(1+\alpha I)^{2} E} \\
\left|B_{21}\right|=\frac{\gamma E}{I}
\end{gathered}
$$

Note that since $B_{11}$ is a scalar, its Lozinskiı̌ measure with respect to any vector norm $\mu_{1}$ is equal to $B_{11}$. Now calculating $\mu_{2}\left(B_{22}\right)$, taking the non-diagonal elements of each columns of $B_{22}$ in absolute value, and then adding to the corresponding columns of the diagonal elements, we have

$$
B_{22}^{\prime}=\left(\begin{array}{cc}
-(\sigma+\mu+\varphi+r)-\frac{\beta S I}{1+\alpha I}+\frac{E^{\prime}}{E}-\frac{I^{\prime}}{I} & -\mu \\
\frac{\beta S I}{1+\alpha I} & -(\gamma+\sigma+\varphi+r+2 \mu)+\frac{E^{\prime}}{E}-\frac{I^{\prime}}{I}
\end{array}\right) \cdots
$$

Take the maximum of the two diagonal elements of $B_{22}^{\prime}$, we have

$$
\begin{aligned}
\mu_{2}\left(B_{22}\right) & =\max \left\{-(\sigma+\mu+\varphi+r)+\frac{E^{\prime}}{E}-\frac{I^{\prime}}{I}, \quad-(\gamma+\sigma+\varphi+r+3 \mu)+\frac{E^{\prime}}{E}-\frac{I^{\prime}}{I}\right\} \\
& =-(\sigma+\mu+\varphi+r)+\frac{E^{\prime}}{E}-\frac{I^{\prime}}{I} \ldots .(37)
\end{aligned}
$$

Therefore, we have

$$
\begin{aligned}
& g_{1}=\mu_{1}\left(B_{11}\right)+\left|B_{12}\right|=\mu_{1}\left(B_{11}\right)=-(\gamma+\mu)-\frac{\beta S I}{1+\alpha I}+\frac{\beta S I}{(1+\alpha I)^{2} E} \\
& g_{2}=\mu_{2}\left(B_{22}\right)+\left|B_{21}\right|=-(\sigma+\mu+\varphi+r)+\frac{E^{\prime}}{E}-\frac{I^{\prime}}{I}+\frac{\gamma E}{I}
\end{aligned}
$$

From the system of equations in (5) we have

$$
\frac{E^{\prime}}{E}=\frac{\beta S I}{(1+\alpha I) E}-(\gamma+\mu) \text { and } \frac{I^{\prime}}{I}=\frac{\gamma E}{I}-(\sigma+\mu+\varphi+r)
$$

Then we have

$$
\begin{aligned}
& g_{1}=\mu_{1}\left(B_{11}\right)=-(\gamma+\mu)-\frac{\beta S I}{1+\alpha I}+\frac{\beta S I}{(1+\alpha I)^{2} E} \leq \frac{\beta S I}{(1+\alpha I) E}-(\gamma+\mu) \\
& g_{2}=-(\sigma+\mu+\varphi+r)+\frac{E^{\prime}}{E}-\frac{I^{\prime}}{I}+\frac{\gamma E}{I}
\end{aligned}
$$

from which we obtain 


$$
g_{1} \leq \frac{E^{\prime}}{E}-\mu \text { and } g_{2}=\frac{E^{\prime}}{E}-\mu \leq \frac{E^{\prime}}{E}-(\mu-\gamma)
$$

Also, we have

$$
\begin{aligned}
\mu(B) & \leq \sup \left\{g_{1}, g_{2}\right\} \\
& \leq \sup \left\{\frac{E^{\prime}}{E}-\mu, \frac{E^{\prime}}{E}-(\mu-\gamma)\right\} \\
& \leq \frac{E^{\prime}}{E}-(\mu-\gamma) \ldots(41)
\end{aligned}
$$

Integrating both sides simultaneously, we have

$$
\frac{1}{t} \int_{0}^{t} \mu(B) d s \leq \frac{1}{t} \ln \frac{E(t)}{E(0)}-(\mu-\gamma),
$$

so

$$
\lim \sup _{t \rightarrow \infty} \sup _{x_{0} \in K} \frac{1}{t} \int_{0}^{t} \mu(B) d s \leq-(\mu-\gamma)<0
$$

by the hypothesis $\mu-\gamma>0$. Thus, by [31], we have that $P^{*}$ is globally asymptotically stable.

We will now analyze the global stability of the endemic steady states when $I^{*}>I_{0}$. After reducing the system of equations in (18) using $R(t)=\frac{A}{\mu}-S(t)-E(t)-I(t)$, we eliminate the $R(t)$ component from the first equation of system (18) to obtain a three-dimensional model.

Theorem 7.3: Let $Q^{*}\left(S^{*}, E^{*}, I^{*}, R^{*}\right)$ be an endemic equilibrium of (4) with $I^{*}>I_{0}$. If $\mu-\gamma>0$ then the endemic equilibrium $Q^{*}$ is globally stable.

Proof: Since $I^{*}>I_{0}, Q^{*}$ is an equilibrium for system (18). The Jacobian matrix of system (18) is given as

$$
J\left(E_{2}^{*}\right)=\left(\begin{array}{ccc}
-\mu-\frac{\beta S I}{1+\alpha I} & 0 & -\frac{\beta S}{(1+\alpha I)^{2}} \\
\frac{\beta S I}{1+\alpha I} & -(\gamma+\mu) & \frac{\beta S}{(1+\alpha I)^{2}} \\
0 & \gamma & -(\sigma+\mu+\varphi)
\end{array}\right) \ldots \ldots
$$

and its second additive matrix is 


$$
J^{[2]}=\left(\begin{array}{ccc}
-\left(\gamma+2 \mu+\frac{\beta S I}{1+\alpha I}\right) & \frac{\beta S}{(1+\alpha I)^{2}} & \frac{\beta S}{(1+\alpha I)^{2}} \\
\gamma & -\left(\sigma+\varphi+2 \mu+\frac{\beta S I}{1+\alpha I}\right) & 0 \\
0 & \frac{\beta S I}{1+\alpha I} & -(\gamma+\sigma+\varphi+2 \mu)
\end{array}\right)
$$

Choose the function $Q=Q(S, E, I)=\operatorname{diag}\left(1, \frac{E}{I}, \frac{E}{I}\right) ;$ then $Q^{-1}=\operatorname{diag}\left(1, \frac{I}{E}, \frac{I}{E}\right)$ and

$$
Q_{f}=\operatorname{diag}\left(0, \frac{E^{\prime} I-I^{\prime} E}{I^{2}}, \frac{E^{\prime} I-I^{\prime} E}{I^{2}}\right) \ldots \ldots \ldots
$$

Then we have

$$
\begin{aligned}
& Q_{f} Q^{-1}=\operatorname{diag}\left(0, \frac{E^{\prime}}{E}-\frac{I^{\prime}}{I}, \frac{E^{\prime}}{E}-\frac{I^{\prime}}{I}\right) \\
& Q J^{[2]} Q^{-1}=\left(\begin{array}{ccc}
-(\gamma+2 \mu)-\frac{\beta S I}{1+\alpha I} & \frac{\beta S I}{(1+\alpha I)^{2} E} & \frac{\beta S I}{(1+\alpha I)^{2} E} \\
\frac{\gamma E}{I} & -(\sigma+\varphi+2 \mu)-\frac{\beta S I}{1+\alpha I} & 0 \\
0 & \frac{\beta S I}{1+\alpha I} & -(\gamma+\sigma+\varphi+2 \mu)
\end{array}\right)
\end{aligned}
$$

The matrix $C=Q_{f} Q^{-1}+Q J^{[2]} Q^{-1}$ can be written in matrix form as

Where

$$
C=\left(\begin{array}{ll}
C_{11} & C_{12} \\
C_{21} & C_{22}
\end{array}\right)
$$

$$
\begin{array}{ccc}
C_{11}=-(\gamma+2 \mu)-\frac{\beta S I}{1+\alpha I}, & C_{12}=\left(\frac{\beta S I}{(1+\alpha I)^{2} E}, \frac{\beta S I}{(1+\alpha I)^{2} E}\right) \\
C_{21}=\left(\frac{\gamma E}{I}, 0\right)^{T}, & \\
C_{22}=\left(\begin{array}{cc}
-(\sigma+\varphi+2 \mu)-\frac{\beta S I}{1+\alpha I}+\frac{E^{\prime}}{E}-\frac{I^{\prime}}{I} & 0 \\
\frac{\beta S I}{1+\alpha I} & -(\gamma+\sigma+\varphi+2 \mu)+\frac{E^{\prime}}{E}-\frac{I^{\prime}}{I}
\end{array}\right)
\end{array}
$$

Let $(u, v, w)$ be a vector in $\mathbb{R}^{3}$, its norm $\|\cdot\|$ is defined as

$$
\|(u, v, w)\|=\max \{|u|,|v|+|w|\} \ldots \ldots(
$$

Let $\mu(C)$ be the Lozinskir measure with respect to this norm. Thus, we choose

$$
\mu(C) \leq \sup \left\{f_{1}, f_{2}\right\}
$$

where $f_{1}=C_{11}+\left|C_{12}\right|$ and $f_{2}=\mu_{2}\left(C_{22}\right)+\left|C_{21}\right| ;\left|C_{12}\right|$ and $\left|C_{21}\right|$ are matrix norms with respect to $l_{1}$ vector norm and $\mu_{2}$ denotes the Lozinskii measure with respect to $l_{1}$ vector norm. Then, 


$$
\begin{gathered}
\mu_{1}\left(C_{11}\right)=-(\gamma+\mu)-\frac{\beta S I}{1+\alpha I} \ldots . .(49) \\
\left|C_{12}\right|=\max \left(\frac{\beta S I}{(1+\alpha I)^{2} E}, \quad \frac{\beta S I}{(1+\alpha I)^{2} E}\right)=\frac{\beta S I}{(1+\alpha I)^{2} E} \\
\left|C_{21}\right|=\frac{\gamma E}{I}
\end{gathered}
$$

Note that since $C_{11}$ is a scalar, its Lozinskiǐ measure with respect to any vector norm $\mu_{1}$ is equal to $C_{11}$. Clearly, to obtain $\mu_{2}\left(C_{22}\right)$, taking the non-diagonal elements of each columns of $C_{22}$ in absolute value, and then add it to the corresponding columns of the diagonal elements, we have

$$
C_{22}^{\prime}=\left(\begin{array}{cc}
-(\sigma+\varphi+2 \mu)-\frac{\beta S I}{1+\alpha I}+\frac{E^{\prime}}{E}-\frac{I^{\prime}}{I} & -\mu \\
\frac{\beta S I}{1+\alpha I} & -(\gamma+\sigma+\varphi+2 \mu)+\frac{E^{\prime}}{E}-\frac{I^{\prime}}{I}
\end{array}\right) \cdots
$$

Take the maximum of the two diagonal elements of $C_{22}^{\prime}$, we have

$$
\begin{gathered}
\mu_{2}\left(C_{22}\right)=\max \left\{-(\sigma+\varphi+2 \mu)+\frac{E^{\prime}}{E}-\frac{I^{\prime}}{I}, \quad-(\gamma+\sigma+\varphi+3 \mu)+\frac{E^{\prime}}{E}-\frac{I^{\prime}}{I}\right\} \\
=-(\sigma+\varphi+2 \mu)+\frac{E^{\prime}}{E}-\frac{I^{\prime}}{I} \ldots .(51)
\end{gathered}
$$

We obtain

$$
\begin{aligned}
& f_{1}=\mu_{1}\left(C_{11}\right)+\left|C_{12}\right|=\mu_{1}\left(C_{11}\right)=-(\gamma+\mu)-\frac{\beta S I}{1+\alpha I}+\frac{\beta S I}{(1+\alpha I)^{2} E} \\
& f_{2}=\mu_{2}\left(C_{22}\right)+\left|C_{21}\right|=-(\sigma+\mu+\varphi)+\frac{E^{\prime}}{E}-\frac{I^{\prime}}{I}+\frac{\gamma E}{I}
\end{aligned}
$$

From the system of equations in (18), we have

$$
\frac{E^{\prime}}{E}=\frac{\beta S I}{(1+\alpha I) E}-(\gamma+\mu) \text { and } \frac{I^{\prime}}{I}=\frac{\gamma E}{I}-(\sigma+\mu+\varphi)
$$

Then we have

$$
\begin{aligned}
& f_{1}=-(\gamma+2 \mu)-\frac{\beta S I}{1+\alpha I}+\frac{\beta S I}{(1+\alpha I)^{2} E} \leq \frac{\beta S I}{(1+\alpha I) E}-(\gamma+\mu) \\
& f_{2}=-(\sigma+\varphi+2 \mu)+\frac{E^{\prime}}{E}-\frac{I^{\prime}}{I}+\frac{\gamma E}{I}=\frac{\gamma E}{I}-(\sigma+\mu+\varphi)-\mu+\frac{E^{\prime}}{E}-\frac{I^{\prime}}{I}
\end{aligned}
$$

From which we obtain

$$
f_{1} \leq \frac{E^{\prime}}{E}-\mu \text { and } f_{2}=\frac{E^{\prime}}{E}-\mu \leq \frac{E^{\prime}}{E}-(\mu-\gamma)
$$

Also, we have 


$$
\begin{aligned}
\mu(C) & \leq \sup \left\{f_{1}, f_{2}\right\} \\
& \leq\left\{\frac{E^{\prime}}{E}-\mu, \frac{E^{\prime}}{E}-(\mu-\gamma)\right\} \\
& \leq \frac{E^{\prime}}{E}-(\mu-\gamma) \ldots(55)
\end{aligned}
$$

Integrating both sides simultaneously, we have

$$
\frac{1}{t} \int_{0}^{t} \mu(B) d s \leq \frac{1}{t} \ln \frac{E(t)}{E(0)}-(\mu-\gamma),
$$

so

$$
\lim \sup _{t \rightarrow \infty} \sup _{x_{0} \in K} \frac{1}{t} \int_{0}^{t} \mu(B) d s \leq-(\mu-\gamma)<0 \ldots \ldots
$$

by the hypothesis $\mu-\gamma>0$. Thus, by [31], we have that $Q^{*}$ is globally asymptotically stable.

\section{Conclusion}

In this paper, we studied the global analysis of an SEIRS epidemic model capturing saturated incidence with treatment response. In the theoretical study of this model, we obtain the basic reproduction number, which explains the dynamic behaviour of the model showing that when $R_{0}$ is less than a certain critical value, which we called $R_{1}$, the disease-free equilibrium is globally asymptotically stable, that is, the disease dies out. We also determine the existence of the local and global stability of the disease-free and endemic equilibria and found that the efficiency of the treatment response such as contact tracing, quarantine, case search, pool testing, advanced medical technologies, increased trained personnel, funding medical research, etc, and strict adherence to inhibitory measures such as personal hygiene, social distancing, stay-at-home orders, etc, deployed in tackling the COVID-19 pandemic determines whether the disease will die out or become endemic. Hence, how long COVID-19 pandemic stays with us depends on how much we are willing to take responsibility as individuals and government. 
medRxiv preprint doi: https://doi.org/10.1101/2020.05.15.20103630; this version posted September 12, 2020. The copyright holder for this preprint (which was not certified by peer review) is the author/funder, who has granted medRxiv a license to display the preprint in perpetuity.

It is made available under a CC-BY-ND 4.0 International license .

\section{References}

[1] World Health Organization Coronavirus disease (COVID-2019) situation reports 2020, accessed on February 23, 2020. [cited 2020 February 23]; Available from:

https://www.who.int/emergencies/diseases/novel-coronavirus-2019/situation-reports.

[2] Peiris J.S.M., Guan Y., Yuen K., Severe Acute Respiratory Syndrome. Nature Med. 10:S88S97, 2004.

[3] Zhou P., Yang XL, Wang XG, Hu B, Zhang L, Zhang W et'al. Discovery of a novel coronavirus associated with recent pneumonia outbreak in human and its potential bat origin. bioRxiv, 2020. doi: https://doi.org/10.1101/2020.01.32.914952.

[4] Zaki AM, Boheemen SV, Bestobroer TM, Osterhaus ADME, Fouchier, RAM. Isolation of a novel coronavirus from a man with pneumonia in Saudi Arabia. N. Engl. J. Med. 367 (19):18141820, 2012;

[5] Riou J, Althaus CL. Pattern of Early Human-to-Human Transmission of Wuhan 2019 Novel Coronavirus (2019 n-CoV), December 2019 to January, 2020. Euro. Surveill. 25 (4):200058, 2020. https://doi.org/10.2807/1560-791ES2020.25.4.2000058

[6] Liu Y, Gayle AA, Wilder-Dmith A, Rocklou J. The Reproduction Number of COVID-19 is Higher Compared to SARS Coronavirus. J. Travel Med. 27 (3), 2020. https://doi.org/10.1093/jtm/taa0021

[7] Wu FZS, Yu B, Chen YM, Wang W, Song ZG, Hu Y, Tao ZW, Tian JH, Pei YY, Yuan ML, Zhang YL, Dai FH, Liu Y, Wang QM, Zhang JJ, Xu L, Holmes EC, Zhang YZ. A Coronavirus Associated with Human Respiratory Disease in China, Nature 579 (7798):265-269, 2020.

[8] Chen Y, Guo Y, Pan Y, Zhao ZL. Structure Analysis of the Receptor Binding of 2019 nCoV. Biochemical and Biophysical Research Communications 525 (1):135-140, 2020.

[9] Kadioglu O, Saeed M, Johannes Greten H, Efferth T. Identification of Novel Compounds Against Three Targets of SARS-CoV-2 Coronavirus by Combined Virtual Screening and Supervised Machine Learning [submitted]. Bull. World Health Organ. E-pub: 21 March, 2020. doi: https://dx.doi.org/10.2471/BLT.20.255.943.

[10] Okhuese, V.A. Mathematical Predictions for COVID-19 as a Global Pandemic. medRxiv preprint, 2020 doi: https://doi.org/10.1101/2020.03.19.20038794.

[11] Adhikari S.P., Meng S, Wu YJ, Mao YP, Ye RX, Wang QZ, Sun C, Sylvia S, Rozelle S, Raat H, Zhou H. Epidemiology, Causes and Clinical Manifestation and Diagnosis, Prevention 
medRxiv preprint doi: https://doi.org/10.1101/2020.05.15.20103630; this version posted September 12, 2020. The copyright holder for this preprint (which was not certified by peer review) is the author/funder, who has granted medRxiv a license to display the preprint in perpetuity.

It is made available under a CC-BY-ND 4.0 International license .

and Control of Coronavirus Disease (COVID-19) During the Early Outbreak Period: a Scoping Review. Infectious Diseases of Poverty. 9:29 (2020). https://doi.org/10.1186/s40249-020-00646$\mathrm{X}$

[12] Wu, J. T., Leung, K., Leung G. M., Nowcasting and Forecasting the Potential Domestic and International Spread of the 2019-nCoV Outbreaks Originating in Wuhan, China: A Modeling Study, The Lancet 395 (10225):689-697. doi:10.1016/s0140-6736(20)30260-9. url: https://doi.org/10.1016\%2Fs0140\%2Fs0140-6736\%6736\%2820\%2930260-9

[13] Imai, N., Cori, A., Dorigatti, I, et'al., Report 3: Transmissibility of 2019-nCoV. Available from:

https://fpmag.net/wp-content/uploads/2020/01/Imperial-2019-nCoV-transmissibility.pdf.

[14] Li, D., Lv, J., Botwin G., Braun J., Cao, W., Li, L., McGovern, D.P. Estimating the Scale of COVID-19 epidemic in the United States: Simulations Based on Air Traffic Directly from Wuhan, China. medRxiv doi:10.1101/2020.03.06.20031880. URL: https://www.medrxiv.otg/content/early/2020/03/12/2020.03.06.20031880

[15] Anastassopoulou, C., Russo, L., Tsakris, A., Siettos, C., Data-based Analysis, Modeling and Forecasting of the COVID-19 Outbreak. PloS One 15 (3): e0230405, 2020. doi: 10.1101/2020.02.11.20022186.

[16] Zhao S., Lin, Q., Ran, J., Musa, S.S., Yang, G., Wang W. Wang, Y., Lou, D., Gao, D., Yang, L., He, D., Wang, M. H., Preliminary Estimation of the Basic Reproduction Number of Novel Coronavirus (2019-nCoV) in China from 2019 to 2020: A Data -driven Analysis in the early phase of the outbreak. Int. J. Infectious Dis. 92:214-217, 2020. doi: 10.1016/j.ijid.2020.01.050.

[17] Remuzzi, A., Remuzzi, G., COVID-19 and Italy: What Next?. The Lancet doi:10.1016./s0140-6736(20)30627-9. URL: $\quad$ https://doi.org/10.1016\%2Fs0140$\underline{6736 \% 2820 \% 2930627-9}$

[18] Ming, W-K., Huang, J., Zhang, C.P.J., Breaking down of the healthcare system: Mathematical Modeling for Controlling the novel coronavirus (2019-nCoV) Outbreak in Wuhan China. bioRxiv, 2020. doi: 10.1101/2020.01.27.922443.

[19] Russo, L., Anastassopoulou, C., Tsakris A., Bifulco, G. N., Campana, E.F., Toraldo, G., Siettos, C., Tracing DAY-ZERO and Forecasting the Fade out of the COVID-19 Outbreak in Lombardy, Italy: A Compartmental Modelling and Numerical Optimization Approach. medRxiv preprint, 2020. doi: https://doi.org/10.1101/2020.03.17.20037689. 
medRxiv preprint doi: https://doi.org/10.1101/2020.05.15.20103630; this version posted September 12, 2020. The copyright holder for this preprint (which was not certified by peer review) is the author/funder, who has granted medRxiv a license to display the preprint in perpetuity.

It is made available under a CC-BY-ND 4.0 International license .

[20] Esteva L., Matias M., A Model for Vector Transmitted Diseases with Saturation Incidence. Journal of Biological Systems, 9(4), pp. 235 - 245, 2001.

[21] Kermack W., McKendrick A. Contributions to the Mathematical Theory of Epidemics. Proc. Roy. Soc. A., 115 (1927) pp. 700 - 712, 1927.

[22] Capasso V., Serio G., A Generalization of the Kermack-McKendrick Deterministic Epidemic Model, Math. Biosciences, Vol. 42, pp. 43 - 61, 1978.

[23] Wang W., Ruan S., Bifurcations in an epidemic model with constant removal rate of incentives. Journal of Mathematical Analysis and Applications. Vol. 291, pp 775 - 793, 2004.

[24] Wang W. Backward Bifurcation of an Epidemic Model with Treatment. Mathematical Biosciences, Vol. 201, pp. 58 - 71, 2006

[25] Zhou L., Fan M. Dynamics of an SIR Model with Limited Medical Resources Revisited. Nonlinear Analysis: Real World Applications, 13 (1), pp. 312 - 324, 2012.

[26] Zhang J., Jia J, Song X. Analysis of an SEIR Epidemic Model with Saturated Incidence and Saturated Treatment Function. Sci. World J. (2014), pp. 1 - 11. Article ID 910421.

[27] Agrawal, A., Tenguria, A., Modi, G. Global Analysis of an SEIRS Epidemic Model with Saturated Incidence and Saturated Treatment. Asian Journal of Mathematics and Computer Research, 22(2): 43-56, 2017.

[28] Badole M., Tiwari S. K., Gupta, V., Agrawal, A. Global Dynamics of an SEIR Epidemic Model with Saturated Incidence Under Treatment. International Journal of Scientific Research in Mathematical and Statistical Sciences 5 (3), pp. 48 - 57, 2018.

[29] Al-Sheikh, S. A. Modeling and analysis of an SEIR Epidemic Model with a Limited Resource for Treatment. Global Journal of Science Frontier Research Mathematics and Decision Science, 12 (4), pp. 57 - 66, 2012.

[30] Anderson R. M., May R. M., Population Biology of Infectious I, Nature 180 (1979) pp. 361 $-367,1979$

[31] Li, M. Y., Muldowney, J. S., A Geometric Approach to Global Stability Problems, SIAM Journal on Mathematical Analysis, 27 (4), pp. 1070 - 1083, 1996.

[32] Van den Driessche, P., Watmough, J. Reproduction Numbers and Subthreshold Endemic Equilibria for Compartmental Models of Disease Transmission. Mathematical Biosciences, 180, $29-48,2002$. 
medRxiv preprint doi: https://doi.org/10.1101/2020.05.15.20103630; this version posted September 12, 2020. The copyright holder for this preprint (which was not certified by peer review) is the author/funder, who has granted medRxiv a license to display the preprint in perpetuity.

It is made available under a CC-BY-ND 4.0 International license .

[33] Acedo L., Gonzalez-Parra G., Arenas A. J. An Exact Global Solution for the Classical Epidemic Model. Nonlinear Anal. RWA 11 (2010) pp. 1819 - 1825.

[34] Hu Z.X., Liu S. Wang H. Backward Bifurcation of an Epidemic Model with Standard Incidence Rate and Treatment Rate. Nonlinear Anal. RWA 9 (2008), pp. 2302 - 2312.

[35] Ma W., Song M., Takeuchi Y., Global Dynamics of a Class of SEIRS Epidemic Model with Time Delay. Appl. Math. Lett. 17 (2004), pp. 1141 - 1145.

[36] Nakata Y., Kuniya T., Global Dynamics of a Class of SEIRS Epidemic Models in a Periodic Environment, J. Math. Anal. Appl. 363 (2010), pp. 230 - 237.

[37] Zhang X, Liu X.-N., Backward bifurcation of an epidemic model with saturated treatment function, Journal of Mathematical Analysis and Applications, vol. 348, no. 1, pp. 433-443, 2008.

[38] Liu J., Bifurcation analysis for a delayed SEIR epidemic model with saturated incidence and saturated treatment function, Journal of Biological Dynamics, 13:1, 461-480, 2019

DOI: 10.1080/17513758.2019.1631965

[39] Pérez, A. G. C., Avila-Vales, E., García-Almeida, G. E., Bifurcation Analysis of an SIR Model with Logistic Growth, Nonlinear Incidence, and Saturated Treatment, Complexity Volume 2019, Article ID 9876013, pp. 21. https://doi.org/10.1155/2019/9876013 